\title{
SYMMETRY CLASSES OF LASING MODES IN TWO NANOSPACED MICRODISKS
}

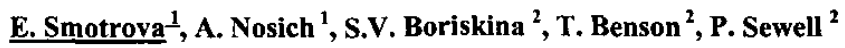 \\ 'Institute of Radiophysics and Electronics, ul. Proskury 12, 61085, Kharkov, Ukraine \\ ${ }^{2} \mathrm{G}$. Green Institute for EM Research, University of Nottingham, NG7 2RD, Nottingham, UK \\ E-mail: elena_smotrova@yahoo.com
}

\begin{abstract}
We present a data on the spectra and thresholds of two optically coupled microdisk lasers. The study is based on the reduction of the $3 D$ lasing eigenvalue problem to a $2 D$ one, through the effective index approximation. The latter problem is formulated in rigorous manner for Maxwell's equations with transmission boundary conditions at the disk boundaries and the radiation condition at infinity.
\end{abstract}

\section{INTRODUCTION}

Semiconductor microdisk lasers attract huge interest in today's dense photonic circuit design as ultra-lowthreshold sources of light [1-3]. Recently, it has been reported that arrays of microdisks coupled in one or two spatial directions can be promising for large-scale applications [4-6]. Although the lasing modes of microdisks are known to demonstrate whispering-gallery effect famous since Lord Rayleigh [7], their features have been studied only with approximate methods $[1,2]$. To quantify both frequency spectra and threshold gains of microcavity lasers, we had introduced new Lasing Eigenvalue Problem (LEP) and studied the lasing in a single microdisk [8]. In this paper, we consider a pair of proximity-coupled microdisks located in the same plane and separated with nanosize air gap, as a simplest microdisk array.

\section{LEP FOR TWO CIRCULAR CAVITIES}

Suppose that two disks (located in the air) have the same thickness $d$ and real-valued refraction index $\alpha$, while their radii are generally different, $a_{1,2}$. Electromagnetic field depends on time as $e^{-i \omega t}$, where $\omega$ is the angular frequency; free-space wavenumber is $k=\omega / c=2 \pi / \lambda$, where $c$ is the light velocity. Quasi-3D modeling of microdisks is based on the so-called effective-index approach, i.e., the assumption that the dependences of the field $\{\boldsymbol{E}, \boldsymbol{H}\}$ on the vertical coordinate $z$ and in-plane coordinates $\boldsymbol{r}=(r, \varphi)$ can be

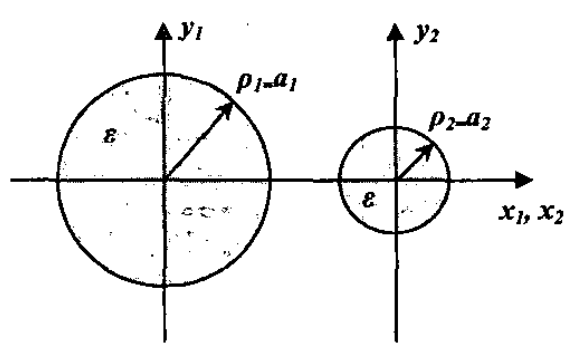

Fig. 1 Geometry of the two-disk problem. separated, e.g., $E_{2}(R)_{=} V_{E}(z) U_{E}(r, \varphi)$ or $H_{z}(R)_{=} V_{H}(z) U_{H}(r, \varphi)$. Although neither boundary conditions on disk surface nor radiation condition at $R=|r, \varphi, z| \rightarrow \infty$ is separable, such an assumption enables one to write independent differential equations for the functions of $z$ and $r$. The first of them, together with the boundary conditions at $z= \pm d / 2$ and $1-D$ condition at infinity, forms a 1-D eigenvalue problem for the parameter $\alpha_{e f f}$ which is a normalized propagation constant of a TE (or $H_{z}$ ) or TM (or $E_{z}$ ) type guided wave of infinite dielectric slab. Then, to approximately account for the third dimension, in the 2D boundary problem for the function $U$ the bulk refraction index $\alpha$ is replaced with its effective value, $\alpha_{e f}$, which is a function of thickness and frequency.

Consider 2-D problem for two circular dielectric cavities (Fig. 1). The optical field can be characterized by a single function $U$, which is $U_{E}$ or $U_{H}$, depending on the polarization. Off the cavity boundaries $U$ must satisfy the Helmholtz equation with the coefficient $k^{2} v^{2}$, where $v=\alpha_{e f f}-i \gamma, \gamma>0$ inside cavities and $v=1$ out of them. At infinity, we impose Sommerfeld's condition of radiation and seek the pairs of real-valued numbers, $\left(\kappa_{s}, \gamma_{s}\right)$, standing for the normalized frequencies of lasing and associated to them thresholds.

In line with separation of variables, we seek the field function as infinite series in local coordinates: 


$$
U(\rho, \varphi)=\left\{\begin{array}{l}
\sum_{p=-\infty}^{\infty} A_{p} J_{p}\left(k \sqrt{\varepsilon} \rho_{1}\right) e^{i p q_{1}}, \rho_{1}<a_{1} \\
\sum_{p=-\infty}^{\infty} B_{p} J_{p}\left(k \sqrt{\varepsilon} \rho_{2}\right) e^{i p \rho_{2}}, \rho_{2}<a_{2} \\
\sum_{p=-\infty}^{\infty} C_{p} H_{p}^{(1)}\left(k \rho_{1}\right) e^{i p \varphi_{1}}+\sum_{p=-\infty}^{\infty} D_{p} H_{p}^{(1)}\left(k \rho_{2}\right) e^{i p \varphi_{2}},\left(\rho_{1}>a_{1}\right) \wedge\left(\rho_{2}>a_{2}\right)
\end{array}\right.
$$

Then the addition theorem for cylindrical functions and the boundary conditions lead to a set of coupled algebraic equations. After using the orthogonality of exponents and excluding some of unknowns we obtain the homogeneous infinite-matrix equation for the modified expansion coefficients, $X=\left(\left\{x_{n}\right\}_{-\infty}^{\infty},\left\{y_{n}\right\}_{-\infty}^{\infty}\right)^{*}$ :

$$
\begin{gathered}
(I+A) X=0, \quad A=\left(\begin{array}{cc}
0 & A^{(1)} \\
A^{(2)} & 0
\end{array}\right), \\
x_{n}=A_{n} / F_{n}\left(\kappa_{1} v\right) / J_{n}\left(\kappa_{1}\right), \quad y_{n}=B_{n} / F_{n}\left(\kappa_{2} v\right) / J_{n}\left(\kappa_{2}\right), \quad \kappa_{1,2}=k a_{1,2} \\
a_{m p}^{(1)}=\frac{a_{2}}{a_{1}} \frac{V_{p}\left(\kappa_{2}, v\right) J_{m}\left(\kappa_{1}\right)}{F_{p}\left(\kappa_{2}\right) J_{p}\left(\kappa_{2}\right)} H_{m-p}^{(1)}\left(\kappa_{1} l\right), \quad a_{m p}^{(2)}=\frac{a_{1}}{a_{2}} \frac{V_{p}\left(\kappa_{1}, v\right) J_{m}\left(\kappa_{2}\right)}{F_{p}\left(\kappa_{1}\right) J_{p}\left(\kappa_{1}\right)} H_{p-m}^{(1)}\left(\kappa_{1} l\right), \\
F_{n}\left(\kappa_{1}\right)=J_{n}\left(\kappa_{i} v\right) H_{n}^{(1)}\left(\kappa_{i}\right)-v J_{n}^{\prime}\left(\kappa_{i} v\right) H_{n}^{(1)}\left(\kappa_{i}\right), \quad V_{m}\left(\kappa_{i}, v\right)=J_{m}\left(\kappa_{i} v\right) J_{m}^{\prime}\left(\kappa_{i}\right)-v J_{m}^{\prime}\left(\kappa_{i} v\right) J_{m}\left(\kappa_{1}\right), i=1,2,
\end{gathered}
$$

where $l=\rho_{12} / a_{1}$, and $\rho_{12}$ is the distance between the centers of resonators.

The Fredholm second-kind nature of $(2)$ follows from the majorant estimation:

$$
\left|a_{m, p}\right|<\text { const } \frac{(|m|+|p|) !}{|m| !|p| !}\left(\frac{1}{l}\right)^{|m|}\left(\frac{a_{2}}{a_{1} l}\right)^{|p|}<\infty, \quad l>1+\frac{a_{2}}{a_{1}}
$$

To determine the pairs $\left(\kappa_{s}, \gamma_{s}\right)$ we search the characteristic numbers of the matrix operator $A$, i.e., the solutions of the set of transcendental equations, $\operatorname{Re}\{\operatorname{det}(I+A(\kappa, \gamma))\}=0, \quad \operatorname{Im}\{\operatorname{det}(I+A(\kappa, \gamma))\}=0$. Here, the Fredholm property of (2) guarantees the convergence of approximate roots, found from truncated determinant equations, to exact ones if the order of truncation is taken progressively larger.

\section{FAMILIES OF THE SYMMETRY}

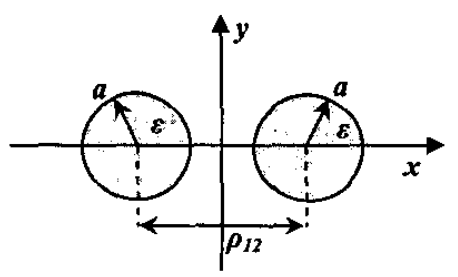

Fig. 2 Two identical circular cavities.

Consider important case of two identical semiconductor resonators. Here all lasing modes can be split into four independent families according to the type of symmetry to the coordinate axes, $x$ and $y$ (see Fig. 2), therefore the set (2) splits into four equations, whose matrix elements are given as

$$
\begin{aligned}
& a_{m p}^{(+, \pm)}= \pm \mu_{p} \frac{J_{m}(\kappa) V_{p}(\kappa, v)}{F_{p}(\kappa, v) J_{p}(\kappa)}\left\{H_{m+p}^{(1)}(\kappa l)+(-1)^{p} H_{m-p}^{(1)}(\kappa l)\right\}, \\
& a_{m p}^{(-, \pm)}= \pm \frac{J_{m}(\kappa) V_{p}(\kappa, \nu)}{F_{p}(\kappa, \nu) J_{p}(\kappa)}\left\{H_{m+p}^{(1)}(\kappa l)-(-1)^{p} H_{m-p}^{(1)}(\kappa l)\right\},
\end{aligned},
$$

where $\mu_{0}=1 / 2, \mu_{n}=1, n>0, m, p=0,1,2, \ldots$ The upper indices indicate the symmetry (anti-symmetry) of mode fields relative to the coordinate axes $x$ and $y$, respectively. Note that after splitting to the independent mode families (7), we have to process effectively twice smaller matrices than with (2), and the algorithm hopping between the roots of different families is eliminated. In computations, we use the secant method for solving truncated determinant equations. 

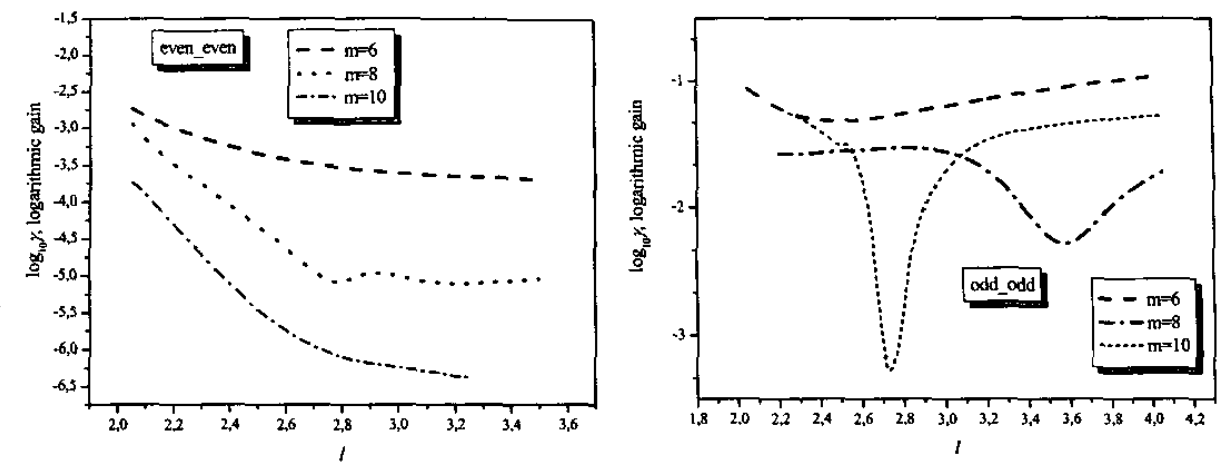

Fig.3. Dependences of threshold gains on the relative distance between two resonators, $\alpha=3.374$.

In Fig. 3, we present the dependences of the thresholds gains of several $\mathrm{WGH}_{\mathrm{m} \text { t }}$ modes (those having $\left|E_{z}\right| \gg>Z_{0}\left|H_{z}\right|$ and $\left.\kappa<m<\kappa \alpha\right)$ on the relative separation between the identical cavities. In this simulation, we considered the E-polarization version of the 2-D LEP. The normalized frequencies of lasing show very little change at the same variation in $l$ and therefore are not shown here. These preliminary results demonstrate that the removal of degeneracy of formerly double degenerate modes of single cavity leads to quite sizable difference in the thresholds although not in the frequencies. In principle, this should be observable in the experiments.

\section{CONCLUSION}

We have presented preliminary results of the cold-cavity-with-gain modeling of a pair of optically coupled identical microdisk lasers. Full-wave analysis shows the proximity effect in the removal of double degeneracy of the WG modes of a single microdisk laser. However, this useful effect is accompanied with a growth of the threshold material gain of the each mode of the doublet.

\section{ACKNOWLEDGEMENT}

Support of the UK-EPSRC to the second author via grant GR/S60693/01(P) is acknowledged with gratitude.

\section{REFERENCES}

[1] R. E. Slusher, A. F. J. Levi, U. Mohideen, S. L. McCall, S. J. Peat-ton, and R. A. Logan, "Threshold characteristics of semiconductor microdisk lasers", Appl. Phys. Lett., vol, 63, no 10, pp. 1310-1312, 1993.

[2] M. Chin, D. Chu, S.-T. Ho, "Estimation of the spontaneous emission factor for microdisk lasers via the approximation of whispering gallery modes", J. Appl. Phys., vol. 75, no. 7, pp. 3302-3307, 1994.

[3] B. Gayral, J.M. Gererd, A. Lemaitre, C. Dupuis, L. Mamin, J.L. Pelouard, "High-Q wet-etched GaAs microdisks containing InAs quantum boxes," Appl. Physics Lett., vol. 75, no 13, pp. 1908- 1910, 1999.

[4] K. Petter, T. Kipp, C. Heyn, D. Heitmann, C. Schuller, "Fabrication of large periodic arrays of AlGaAs microdisks by laser-interference lithography and selective etching," Appl. Phys. Lett., vol. 81, no. 4, pp. 592-595, 2002.

[5] H.W. Choi, C.W. Jeon, M.D. Dawson, "InGaN microring light-emitting diodes," IEEE Photonics Technology Lett, vol. 16, no. 1, pp. 33-35, 2004.

[6] S.J. Choi, Z. Peng, Q. Yang, S.J. Choi, P.D. Dapkus, "Eight-channel microdisk CW laser arrays vertically coupled to common output bus waveguide," IEEE Photonics Technology Lett., vol. 16, no. 2, pp. 356-358, 2004.

[7] Lord Rayleigh, "The problem of the whispering gallery," in Scientific Papers, Cambridge: Cambridge University, vol. 5, pp. 617-620, 1912.

[8] E.I. Smotrova, A.I. Nosich, "Mathematical study of the 2D lasing problem for the whispering-gallery modes in a circular dielectric microcavity" Optical and Quantum Electronics, vol. 36, no. 1/3, pp. 213-221, 2004. 